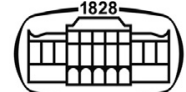

AKADÉMIAI KIADÓ

\title{
Magnetic braking method development for dynamic applications
}

\author{
Laszlo Kazup* (๑) and Angela Varadine Szarka
}

\section{Pollack Periodica \\ An International Journal \\ for Engineering and Information Sciences}

16 (2021) 1, 1-6

DOI:

10.1556/606.2020.00153

(c) 2020 The Author(s)

\section{ORIGINAL RESEARCH}

\section{PAPER}

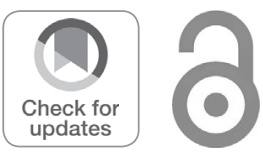

*Corresponding author. E-mail: laszlo.kazup@eiki.hu
Research Institute of Electronics and Information Technology, University of Miskolc, Egyetemváros, H-3515 Miskolc, Hungary

Received: December 31, 2019 • Revised manuscript received: April 18, 2020 • Accepted: June 4, 2020 Published online: March 1, 2021

\section{ABSTRACT}

This paper introduces research on magnetic fields with special attention to developing a new method for braking fast-changing alternating movements. This work is part of a research project aiming to find the most efficient and accurate method for development of linear magnetic brake for dynamic tests in industrial applications. In applications requiring precisely defined and generated characteristics of the braking force, highly reliable and accurate function between the braking force and the controlling current should be investigated.

The goal of this research is to develop accurate and reliable control methods for fast changing magnetic fields used in automatic test solutions of different devices and tools, which have been tested manually before.

\section{KEYWORDS}

linear braking methods, magnetic braking, electromagnetic, brake

\section{INTRODUCTION}

In industry there are lots of automated test methods nowadays. However, fully automated testing is not yet solved in some areas, also in the production of different handheld power tools. For example, performing an automatic lifetime test for reciprocating saws is quite difficult due to the lack of special testers for braking fast-changing alternating movements. The goal of this research is to study fast changing magnetic fields, to develop control methods as well as constructions, which can solve this problem.

\subsection{The first prototype}

A prototype for performing high braking force to load reciprocating saws and similar power tools has been built in 2009, in the early stage of this research. This prototype was based on the construction of voice coil type linear motors, but the stator was excited by a still coil instead of permanent magnets. The structure of this prototype can be seen in Fig. 1.

Theoretical basis of this prototype was evaluation of the airgap's induction distribution and the maximum cutting force by simulation. In addition, experiences of the first prototype provided useful results and information for further development of this construction [1].

First evaluations were implemented in Finite Element Method software Finite Element Method Magnetics (FEMM, open-source simulation software for electric and magnetic problems). Figures 2 and 3 show distribution of induction in the air gap. The major advantage of this construction is a relatively high peak force production in the bottom dead center as the highest cutting force occurs in that position during the operation of jigsaws [1]. However, the spool of the moving coil is a closed aluminum cylinder. Therefore braking force depends on both the moving coil current and the induced eddy current. That is why the range of controlled braking force is limited and its range decreases by increasing the moving speed. It results that the maximum static force is only $390 \mathrm{~N}$ (at 2.5 A moving coil current 


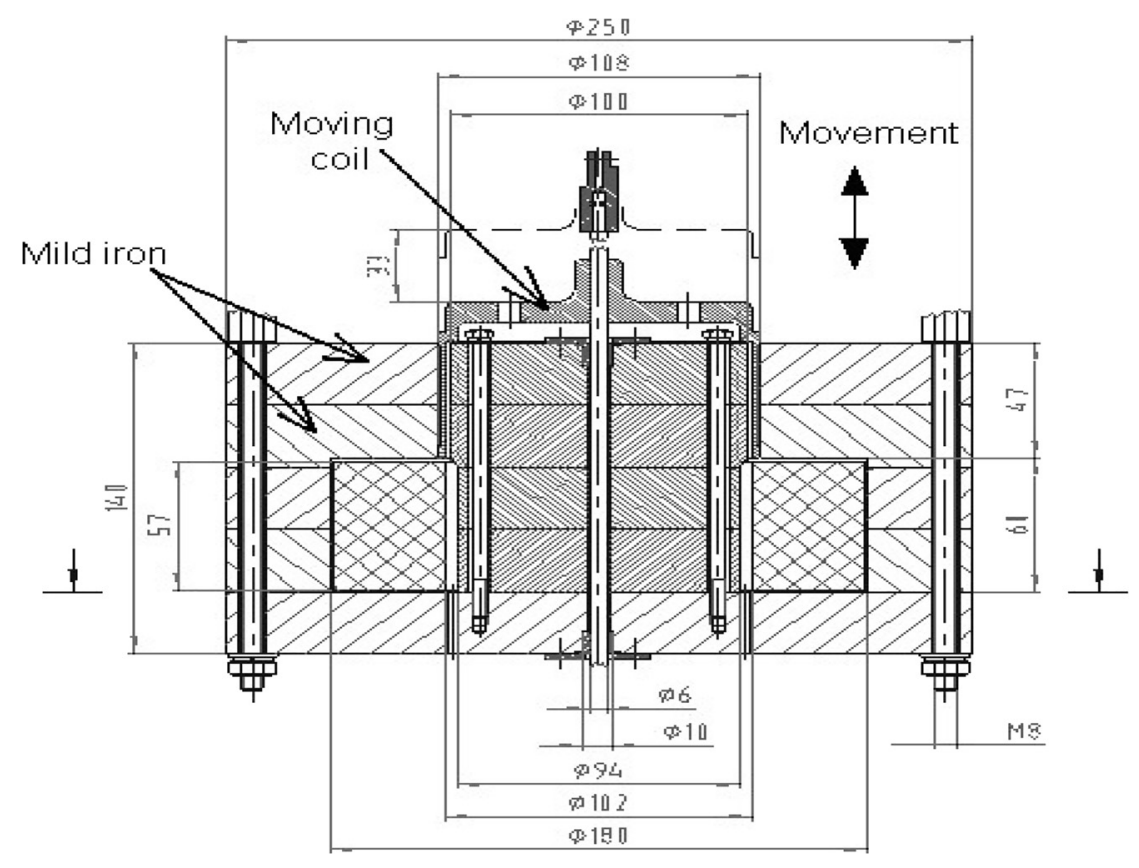

Fig. 1. The first prototype

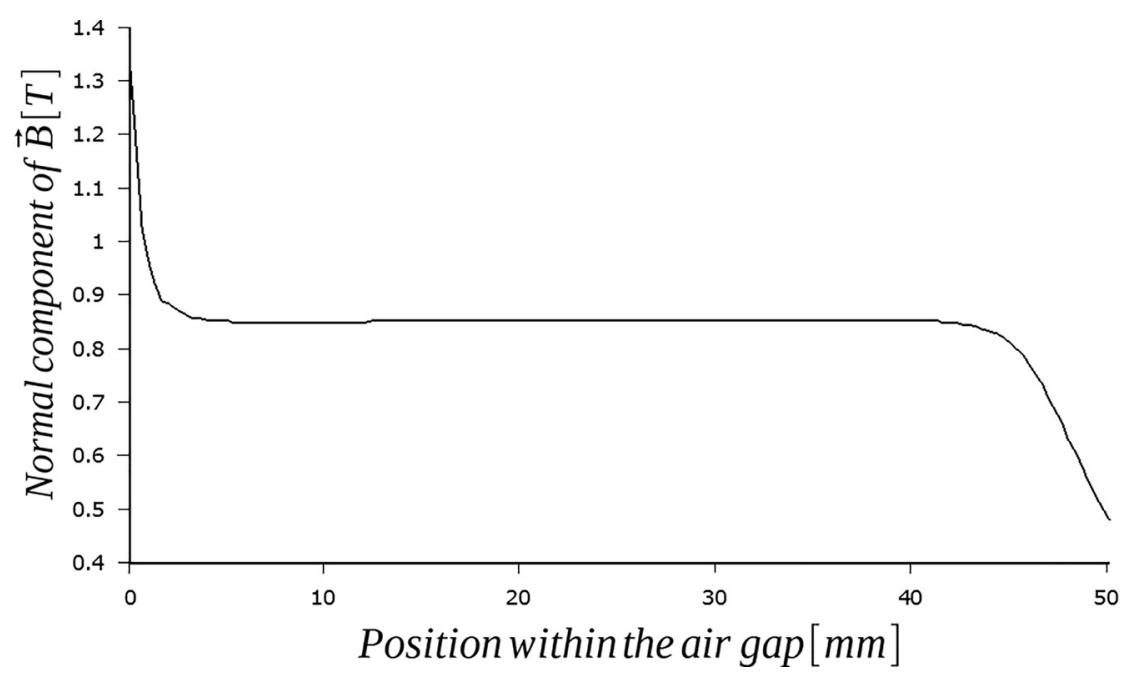

Fig. 2. Air gap induction's distribution of the first prototype - simulation (rectangular plot)

and 3.5 A still coil current), but the dynamic peak force can be much greater due to the eddy current effect. If the excitation of the moving coil is opposite and its summarized magnetic field is larger than the field of eddy currents, then the resultant force can be smaller, but the heat dissipation is quite significant in this case [2-4].

\subsection{Validation of simulation by measurement}

After simulation a measuring system was built to validate the results of the simulation. The main goal of this measurement was to check the accuracy of different model parameters including material settings and boundary conditions. The main part of the measuring system was a gauss meter with thin magnetic probe. During the measurement the probe was moved manually from the bottom of the air gap to its top position, and the flux density was measured in each millimeter [5]. The measuring system and the results can be seen in Figs 4 and 5. The difference between simulated and measured values (average flux densities) is relatively small. The negative peak was occurred by the connections of the steel rings of the stator (in the model the stator is a solid steel volume, but in the real life, producing a similar shape as a solid body with ability of place windings is very difficult). These small differences mean that the material properties and the boundary conditions are correct, and they can be used in further simulations [6].

\subsection{Static force simulation of the first prototype}

Specification of the first prototype defines maximum braking force in measure of $500 \mathrm{~N}$. However, the continuous 


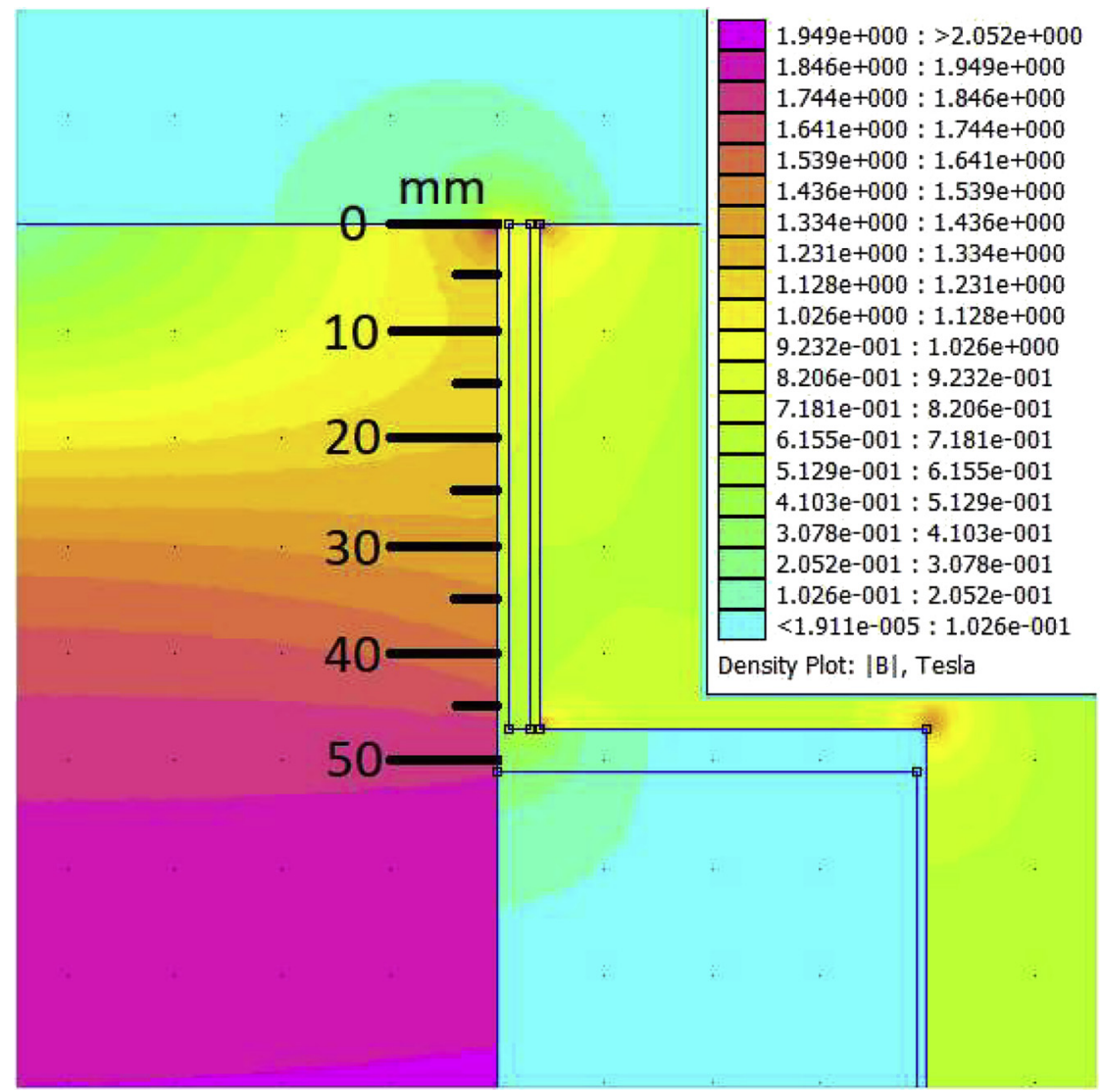

Fig. 3. Air gap induction's distribution of the first prototype - XY distribution

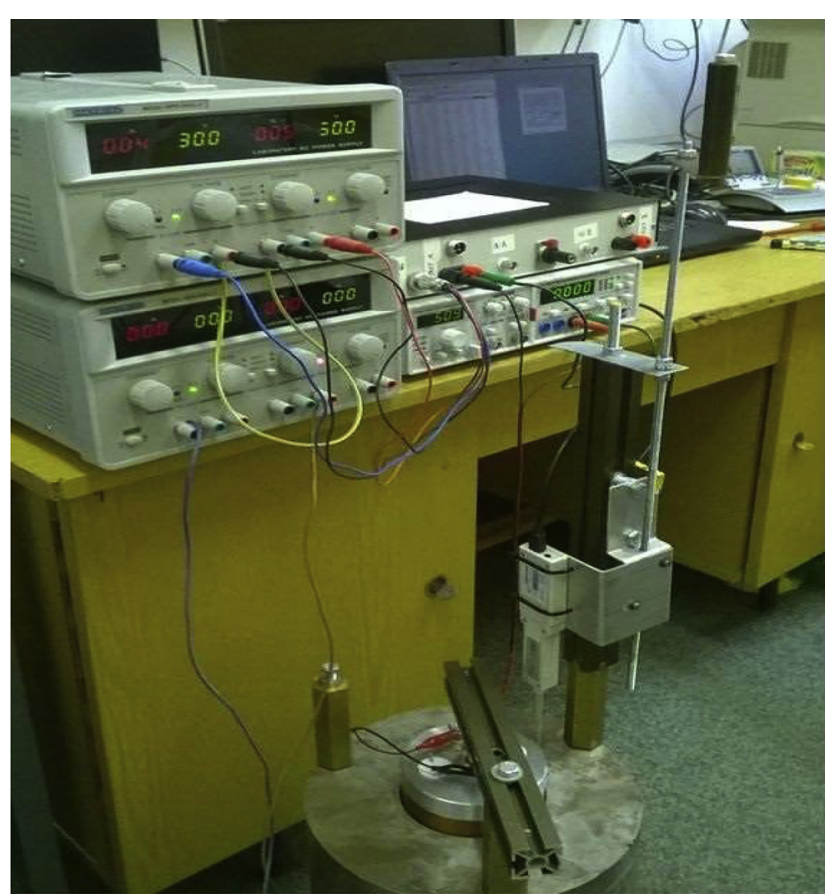

Fig. 4. The induction measurement setup

current in both copper coils is maximized as well (2 A maximum moving coil current and 3.5 A maximum still coil current). Therefore, the current parameters of the force characteristic simulation should conform to the specification. Result of the force simulation is shown in Fig. 6. This simulation was solved by using the ANSYS Maxwell software pack. The maximum static force is lower than the required value (about $260 \mathrm{~N}$ ), but the maximum dynamic force can be significantly greater than this value. Furthermore, the eddy currents in the closed aluminum cylinder help to increase the maximum dynamic force [3].

\section{MORE AIR GAPS, MORE POWER}

\subsection{Multi air-gapped design using permanent magnets}

The first possible way to improve the relatively poor efficiency of the first prototype is applying powerful permanent magnets ( $\mathrm{NdFeB}$ magnets). However, in this case overall construction should be modified. If the buildup includes more permanent magnets, more air gap can be realized. This solution is similar to the construction of industrial voice coil linear motors, but the internal and external steel parts are connected to each other through the air gaps only. This buildup results more difficult mechanical design. The 3-dimension model feasible design can be seen in Fig. 7 .

The average air gap induction is lower than the same value in the first prototype, but the maximum continuous force is $297 \mathrm{~N}$, which value is greater than the same 


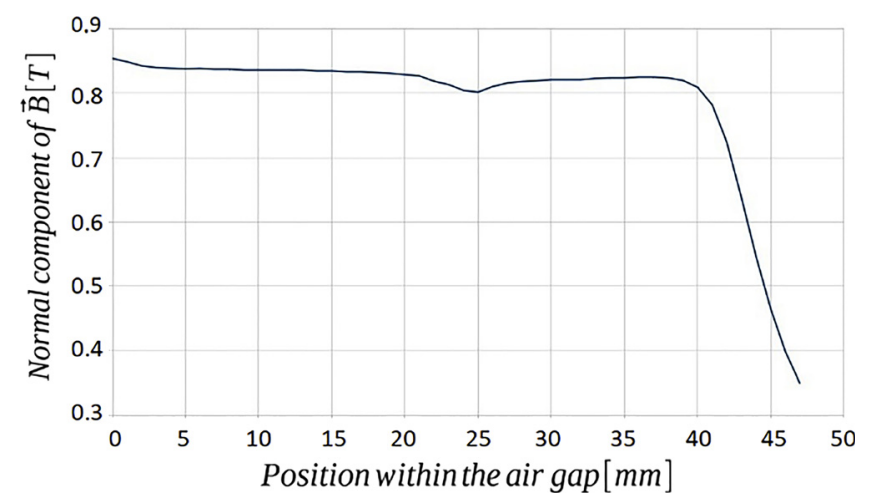

Fig. 5. Induction measurement results

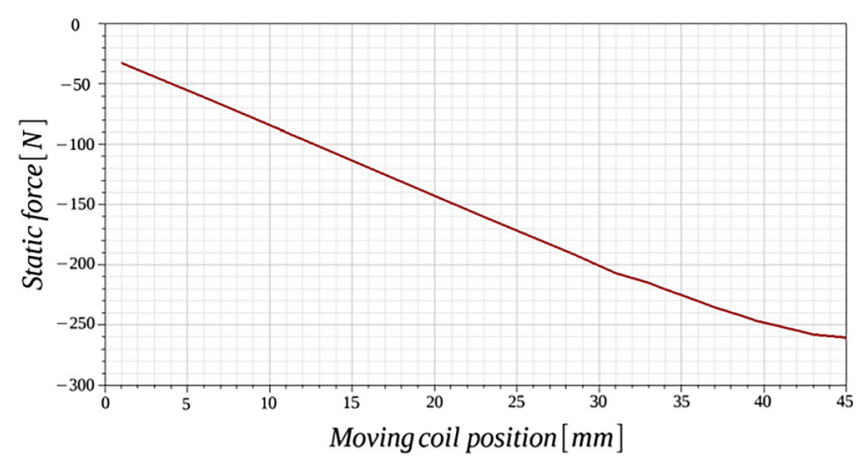

Fig. 6. Force characteristic of the first prototype

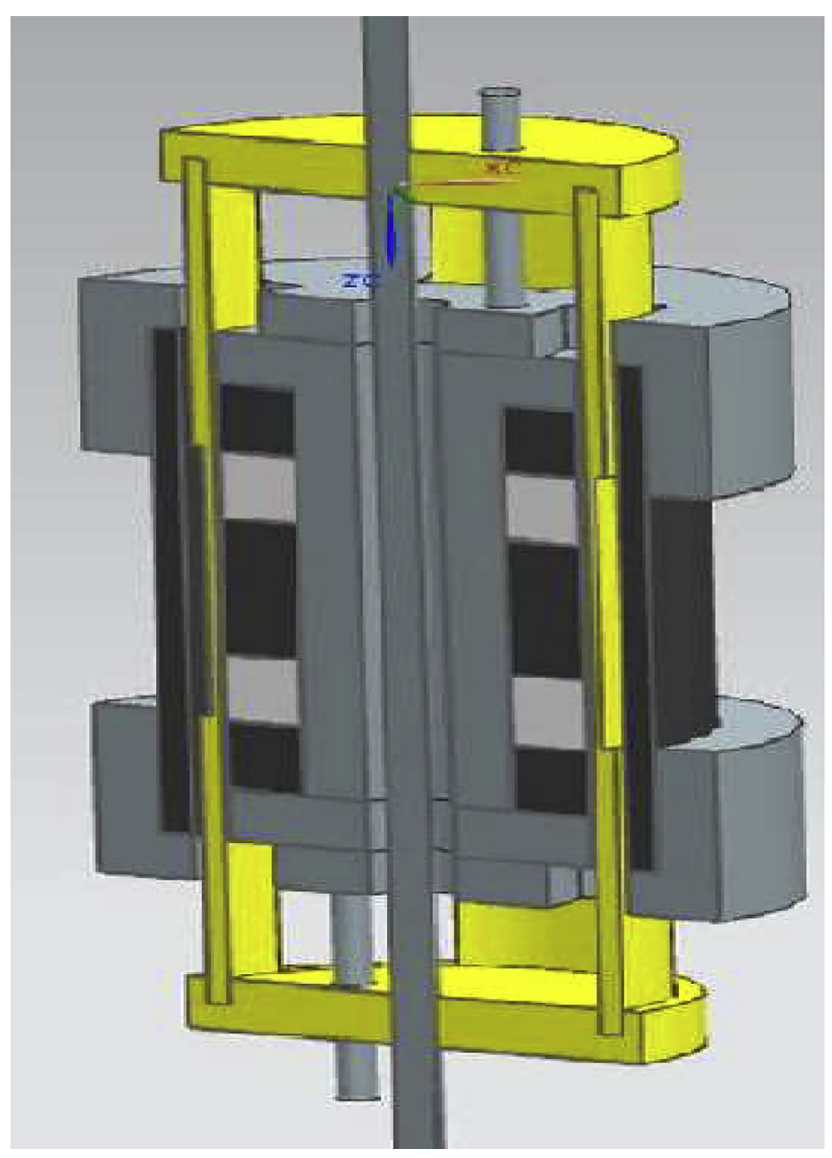

Fig. 7. Multi air gap design using permanent magnets parameter of the previous construction. Furthermore, this solution can produce this force with much lower power dissipation (105 W vs. ca. $300 \mathrm{~W}$ ) thanks to the strong permanent magnets. Due to the symmetric force characteristic the compensation of the moving mass can be simpler. Thanks to the non-conductive coil spool, the momentary braking force can be controlled more precisely; the difference between the static and the dynamic force is small. By using this design, complex force characteristics can be realized within a single moving period and the peak force can be even 1,200 $\mathrm{N}$ (with 1/16 duty cycle max. by using composite, non-conductive spool material with high tensile

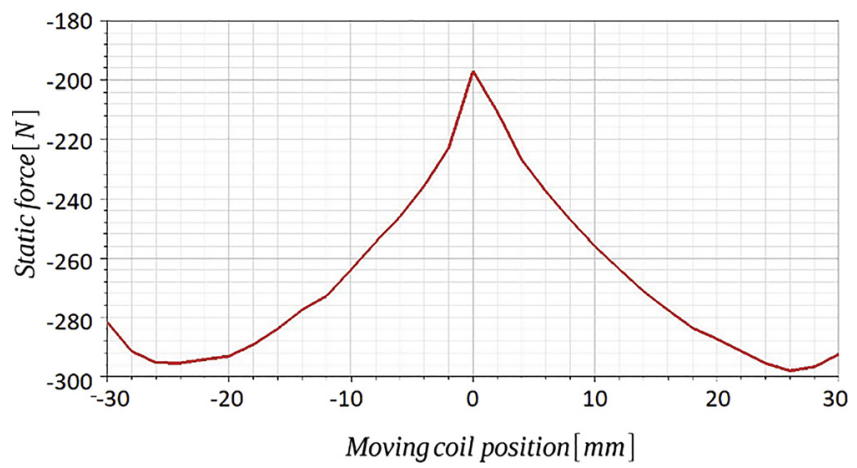

Fig. 8. The simulated static braking force

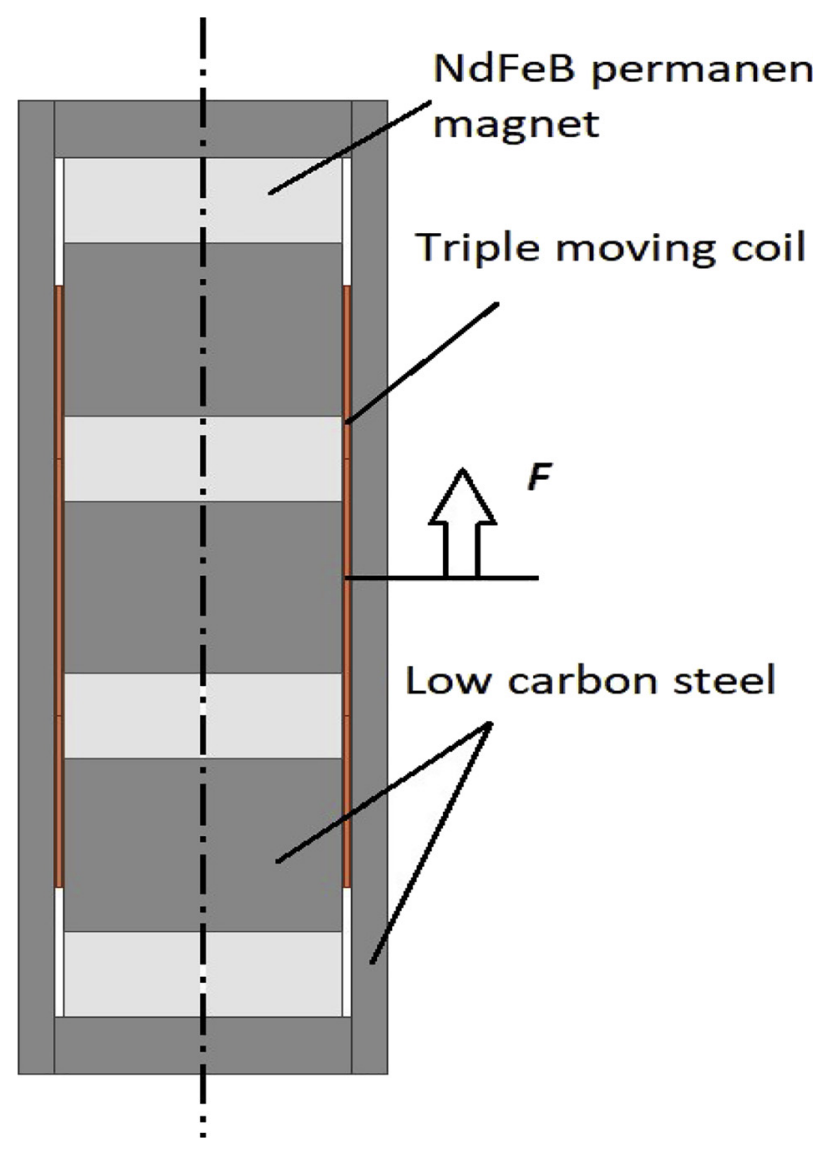

Fig. 9. Multi air gap construction with 4 neodymium permanent magnets 
strength). The results of the force simulation can be seen in Fig. 8.

As the goal of the design is maximization of the braking force, more permanent magnets can be used to increase number of air gaps. More air gaps can handle more moving coils, so the force can be increased as well. In case of appropriate design, the force/power ratio can also be improved. The half cross-section of a possible design (simulation model) is shown in Fig. 9 and the results of the force simulation are shown in Fig. 10. Each curve represents different current direction. Difference between the curves is occurred by the distortion effect of the magnetic field in the moving coil $[7,8]$.

\subsection{Multi air-gapped design using exciter coil}

The NdFeB permanent magnets are robust, but in case of a long-time performance test the temperature can be

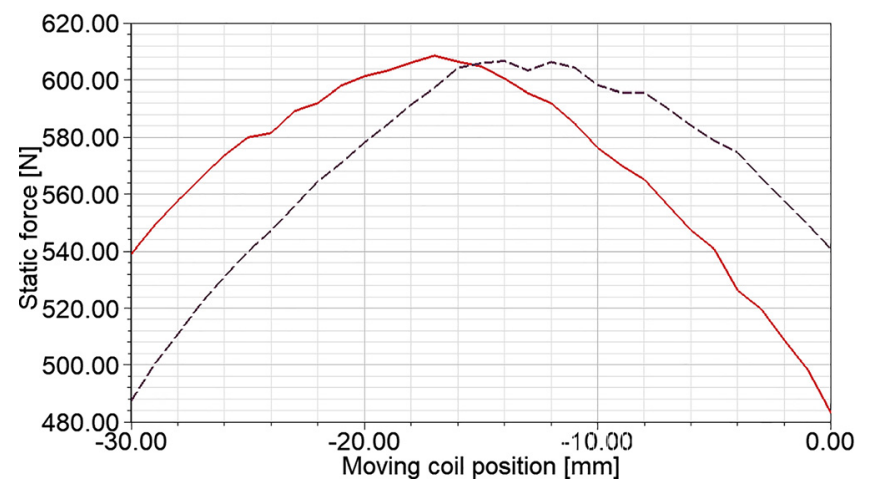

Fig. 10. Force simulation results of the construction of Fig. 7

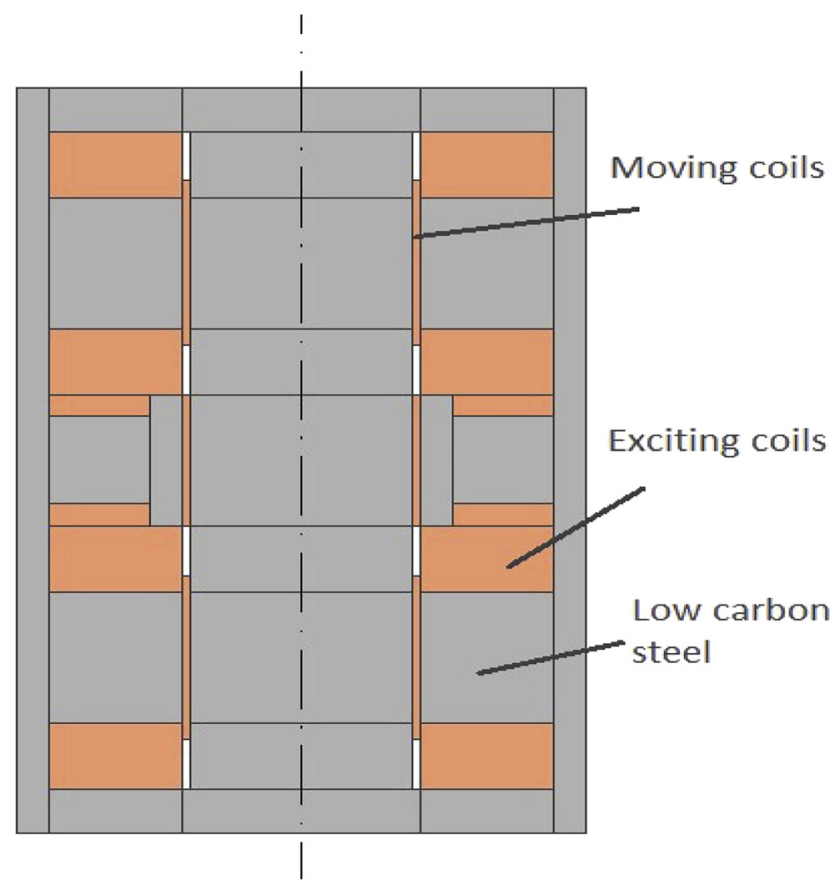

Fig. 11. Multi air gap design with exciter coil

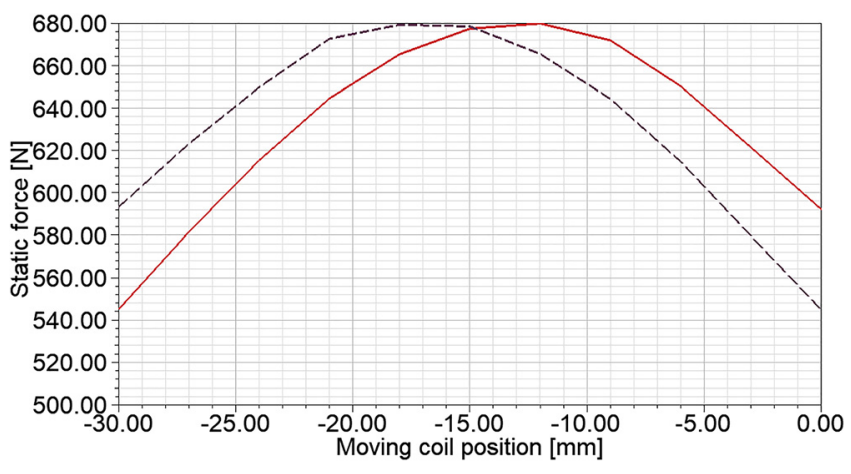

Fig. 12. Multi air gap design with exciter coil-force simulation results

continuously high; therefore design using exciting coils can be reliable in these cases $[9,10]$.

Figure 11 shows the simulation model of a multi air-gap construction using exciter coils. The physical dimensions and the power dissipation increase, however, this construction is not so sensitive to long term high temperatures. But in case of longtime operation, high power testing is essential for determination of the proper cooling. The simulated static force can be seen in Fig. 12. The dashed curve shows the result in case of negative moving coil current. The difference between the curves is occurred by the air gap field distortion.

\section{CONCLUSION}

Using strong permanent magnets efficiency of the system can be maximized, and the mass can be minimized, however in this case long term high temperature conditions should be avoided. In field of industrial lifetime testing design with exciter coils seems to be a reliable solution. From the other side, major disadvantage of this solution is the weaker dynamic behavior and the higher eddy current loss. The preliminary dynamic simulations show that reluctance of the magnetic circuit is smaller and inductance of the moving coil is not a linear function of the current because of the saturated parts of the soft steel core.

Results of simulations show that traditional "speakertype" magnetic circuit is inefficient in this application field due to the relatively high flux leakage. Magnetic circuits including two or more magnets and three or more air-gaps can provide higher gap induction and concentrated flux of two magnetic circuits into one air gap. However mechanical construction is more complex in this case.

\section{REFERENCES}

[1] A. Váradiné Szarka, "Linear magnetic break of special test requirements with dynamic performance," J. Electr. Electron. Eng., vol. 3, no. 2, pp. 237-240, 2010. 
[2] C. H. Chen, A. K. Higgins, and R. M. Strnat, "Effect of geometry on magnetization distortion in closed-circuit magnetic measurements," J. Magnet. Magn. Mater., vol. 320, no. 9, pp. 1597-1656, 2008.

[3] D. Joneit, N. Nadler, and H. Heuer, "Correction of eddy current measurements to obtain accordance with simulation results," NDT\&E Int., vol. 62, pp. 167-171, 2014.

[4] G. Betta, L. Ferrigno, M. Laracca, P. Burrascano, M. Ricci, and G. Silipigni, "An experimental comparison of multi-frequency and chirp excitations for eddy current testing on thin defects," Measurement, vol. 63, pp. 207-220, 2015.

[5] D. Martien, "Introduction to AC susceptibility," AC Magnetic Measurements, Application Note. USA: Quantum Design, 2001.
[6] H. E. Knoepfel, Magnetic Fields: A Comprehensive Theoretical Treatise for Practical Use. John Wiley \& Sons, 2008.

[7] L. Kazup and A. Váradiné Szarka, "Diagnostics of air-gap induction's distortion in linear magnetic brake for dynamic applications," in XXI IMEKO World Congress on Measurement in Research and Industry, Prague, Czech Republic, Aug. 30-Sept. 4, 2015, 2015, pp. 905-908.

[8] M. Kuczmann, "Nonlinear finite element method in magnetism," Pollack Periodica, vol. 4, no. 2, pp. 13-24, 2009.

[9] G. Kovács and M. Kuczmann, "Simulation of a developed magnetic flux leakage method," Pollack Periodica, vol. 4, no. 2, pp. 45-56, 2009.

[10] N. A. Spaldin, Magnetic Materials: Fundamentals and Device Applications. Cambridge University Press, 2003. 